\title{
Carte d'assuré: que pense, que fait la FMH?
}

La future carte d'assuré, exigée par le Parlement par le biais d'une modification de la LAMal (art. 42a) en 2004, crée manifestement beaucoup d'inquiétude dans le corps médical; les lettres de lecteur publiées dans le BMS en témoignent, mais la correspondance que reçoit directement la FMH ainsi que de nombreuses interventions individuelles auprès des autorités fédérales le montrent aussi.

Ces inquiétudes sont justifiées et sous-tendent également nos propres interventions; il règne cependant auprès des médecins une certaine confusion dans ce dossier, et quelques notions doivent être précisées:

- effectivement, une carte d'assuré doit être introduite en 2008 ou 2009, la loi l'exige, comme elle exige aussi que cette carte soit «utilisée pour la facturation des prestations» relevant de la LAMal (art. 42a, al. 2);

- cette carte d'assuré ne doit comporter que des données administratives, il ne s'agit en aucun cas d'une carte de santé, d'un dossier médical électronique ou de quoi que ce soit de ce genre;

- que quelques données médicales «d'urgence» puissent figurer sur la carte d'assuré est une option laissée au libre choix de chaque utilisateur (art. 42a, al. 4), il n'y peut y avoir là aucune obligation;

- une vraie carte de santé serait un projet totalement différent, d'un tout autre ordre de grandeur, exigeant des décisions politiques et un financement spécifiques; même si la carte d'assuré est conçue pour pouvoir le cas échéant, dans le futur, servir de support à la carte de santé, un glissement subreptice d'un type de carte vers un autre n'est donc pas à craindre.

Cela dit, et même s'il ne s'agit que d'une «simple» carte d'assuré, nous nous trouvons ainsi, comme médecins, face à une obligation légale qui peut éventuellement avoir des répercussions positives dans notre pratique quotidienne, mais aussi des conséquences extrêmement lourdes et difficiles.

Il doit donc être clair que si, en principe, nous ne sommes pas contre une carte d'assuré, nous nous opposerons par contre avec la dernière énergie à ce qu'elle soit introduite sans tenir compte des exigences liées à une gestion raisonnable de nos cabinets et à un système de santé respectueux de tous ses partenaires - y compris les patient-es, et les médecins.

Et cela, l'administration fédérale en charge de ce dossier doit le comprendre; elle doit apprendre à écouter et à respecter la voix du corps médical, directement concerné par ce projet; elle doit comprendre aussi que nous considérons ce dossier comme un test important de l'esprit de collaboration qu'on peut attendre d'elle.

Concrètement, nous n'avons évidemment rien contre la simplification des tâches administratives. Par contre, assumer des coûts d'équipement qui reviennent à d'autres, accepter de nouvelles obligations, lourdes, au niveau des connexions informatiques et internet des cabinets, menacer le fonctionnement des trust-centers ou de la Caisse des médecins, pousser subrepticement à la généralisation du tiers payant, mettre en danger le secret médical, et surtout, alourdir de façon insupportable le fonctionnement de nos secrétariats, n'entre pas en ligne de compte.

Le projet d'ordonnance introduisant la carte d'assuré est déjà en consultation interne dans l'administration fédérale et sera soumis durant l'automne aux associations concernées. Nous craignons beaucoup que malgré notre participation active et ouverte, constructive, aux travaux ayant mené à son élaboration, ce projet d'ordonnance ne réponde de loin pas à nos exigences; il faudra donc que l'administration fédérale assume son rôle en prenant soigneusement en compte les réponses que nous ne manquerons pas de faire lors de cette consultation.

Faute de quoi nous sommes prêts à prendre toutes les mesures nécessaires pour être entendus et respectés, dans ce dossier.

Jacques de Haller, Président de la FMH 